\title{
Head and Neck Manifestations of Granulomatosis with Polyangiitis: A Retrospective analysis of 19 Patients and Review of the Literature
}

\author{
Claudio Carnevale ${ }^{1}$ Diego Arancibia-Tagle ${ }^{2}$ Pedro Sarría-Echegaray ${ }^{1} \quad$ Guillermo Til-Pérez ${ }^{1}$ \\ Manuel Tomás-Barberán²
}

${ }^{1}$ Department of Otorhinolaryngology, Hospital Universitari Son Espases, Palma de Mallorca, Illes Balears, Spain

2 Department of Otorhinolaryngology and Head and Neck Surgery, Hospital Universitari Son Espases, Palma de Mallorca, Illes Balears, Spain

\begin{abstract}
Address for correspondence Claudio Carnevale, MD , Department of Otorrinolaringología, Hospital Universitari Son Espases, Carretera Valldemossa 79, Palma de Mallorca, Islas Baleares 07120, Spain (e-mail: claudio.carnevale.orl@gmail.com).
\end{abstract}

Int Arch Otorhinolaryngol 2019;23:165-171.

\begin{abstract}
Keywords

- granulomatosis with polyangiitis

- vasculitis

- anti-neutrophil cytoplasmic antibodies

Introduction Granulomatosis with Polyangiitis (GPA) is a small vessel vasculitis characterized by a necrositing granulomatous inflammation of the upper and lower respiratory tracts and focal/proliferative glomerulonephritis. In more than $70 \%$ of the cases, the presenting symptoms are head and neck manifestations that are often misdiagnosed as infectious or allergic in etiology.

Objective The present study provides an analysis of head and neck manifestations in a series of patients diagnosed with GPA. It also evaluates their medical and surgical treatment and provides a review of the relevant literature.

Methods A retrospective analysis of 19 patients diagnosed with GPA at a public tertiary care hospital between 2006 and 2017 was performed.

Results A total of 19 patients were included in the present study, and 16 of them presented head and neck manifestations. Sinonasal symptoms were the most common, affecting $56 \%$ of the patients, followed by laryngotracheal (31.25\%) and ear (25\%) symptoms. In 7 patients, sinonasal symptoms were the first manifestation of the disease (43.75\%). Four patients underwent surgery at some stage of the disease.

Conclusions Head and neck involvement is common in GPA and may stand for the first or the only manifestation of the disease. The otolaryngologists play a central role in the diagnosis and long-term treatment of these patients, and they have to keep this pathology in mind when treating patients with ENT symptoms that do not respond as expected to the treatment.
\end{abstract}

\section{Introduction}

Granulomatosis with polyangiitis (GPA), commonly known as Wegener granulomatosis (GPA) is a systemic autoimmune disease of unknown etiology, characterized in its fullblown manifestation by a necrotising granulomatous inflammation of the upper and lower respiratory tracts, in addition to focal or proliferative glomerulonephritis. Systemic vasculitis affects small to medium-sized vessels. ${ }^{1}$ Classically, GPA involves a triad of organ systems: the upper respiratory tract, the lungs, and the kidneys, but not all of the patients present with involvement of all the areas, and limited forms of GPA confined only to the head and to the neck are not uncommon. Therefore, both limited and received

November 26, 2017

accepted

September 10, 2018

published online

March 1, 2019
DOI https://doi.org/

10.1055/s-0038-1675759. ISSN 1809-9777.
Copyright $\odot 2019$ by Thieme Revinter

Publicações Ltda, Rio de Janeiro, Brazil
License terms

c) $\oplus \triangleq \$$ 
systemic varieties of the disease can exist, including the following:

- Head and neck alone. Head and neck symptoms are often the first and only manifestation of this pathology

- Head and neck and lungs

- Head and neck, lungs, and kidneys ${ }^{2,3}$

More than $70 \%$ of the symptoms can be found under the appearance of nasal, sinus, ear, or tracheal manifestations, and upper respiratory tract involvement generally precedes pulmonary or renal involvement. The otolaryngologic symptoms of GPA are often misdiagnosed as infectious or allergic in etiology. ${ }^{4}$ The prevalence of GPA is estimated at 3 cases per 100,000 inhabitants in the U.S.A. and at 16 per 100,000 inhabitants in Southern Sweden. Recent series have shown that more than $90 \%$ of all GPA patients are white, while African-Americans, Hispanics and Asians together represent only between 1 and $4 \%$ of the cases. A collaborative European study suggested that the incidence of GPA has a North-South gradient, with a higher incidence in Scandinavian countries and a relatively lower incidence in southern Europe. ${ }^{5}$ The most common ages at presentation are the sixth and seventh decades of life, but GPA can arise at any age (several cases of affected children have been described), and males and females are similarly affected. ${ }^{6}$ The precise etiology of GPA remains obscure, but it seems to be autoimmune in nature. Antineutrophil cytoplasmic antibody (c-ANCA) is a serologic hallmark of GPA. It has been hypothesized that c-ANCA may be formed against complementary peptide sequences to proteinase-3 (PR3). These antisense sequences may be endogenous or introduced via an exogenous infectious pathogen. The pathogen most specifically implicated is Staphylococcus aureus, which exhibits a protein with a peptide sequence that mimics that of the antisense proteinase 3 (PR3). ${ }^{7}$ The circulating antibodies to the antisense PR3 sequences then cross-react with PR3 peptide sequences, thereby functioning as autoantibodies. In vitro studies have shown that antineutrophil antibodies (ANCA) exert their pathogenic effect by binding to and activating neutrophils, which causes the release of oxygen radicals, lytic enzymes, and inflammatory cytokines. Antineutrophil antibodies may also induce immune complex formation and may directly adhere to and kill vascular endothelial cells, resulting in vasculitis. ${ }^{8}$ Head and neck manifestations of GPA account for the initial presenting symptoms in $73 \%$ of the patients, who are ultimately diagnosed with GPA. ${ }^{9}$ The problem is that the symptoms are often non-specific, such as external or middle otitis, nasal obstruction, and rhinorrhea, so the diagnosis of GPA is often difficult. This obviously causes delay concerning the confirmation of the diagnosis and the beginning of treatment, which results in significant sequelae secondary to tissue destruction in the affected areas of the head and the neck. The aim of the present article is to analyze the characteristics of patients diagnosed with GPA at our hospital in the past 11 years, to describe the most common head and neck symptoms that are often the first or the only clinical manifestations of this pathology, and to review the medical literature.

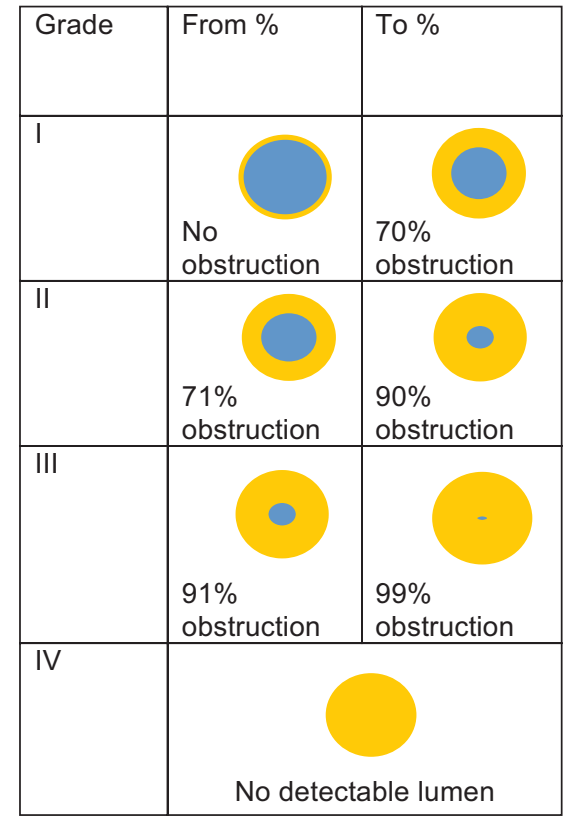

Fig. 1 Myer-Cotton classification.

\section{Methods}

We have conducted a retrospective study including patients diagnosed with GPA at a public tertiary care hospital in the region of Balearic Islands, Spain, between 2006 and 2017. We have reviewed the medical records of the patients and collected the main clinical data associated with head and neck involvement. The histopathological criteria of GPA are the presence of necrotising granulomatous vasculitis affecting the arterial vessel walls or the perivascular or extravascular region. The diagnosis of GPA was based on the presence of compatible clinical lesions associated with the histological confirmation of GPA or positive ANCA. In the seven cases in which nasosinusal symptoms were the first or only manifestation of the disease, a nasal mucosa biopsy was performed and confirmed the diagnosis in five of them. An indirect immunofluorescence was performed in all patients to confirm the presence of antineutrophil cytoplasmic antibodies (c-ANCA) or of antineutrophil perinuclear antibodies (p-ANCA). Regarding the laryngotracheal involvement, we have used the MyerCotton classification ( - Fig. 1) to classify the grade of subglottic stenosis based on the direct visualization of the transversal diameter of the stenosis in a high definition laryngeal videoendoscopy. To measure the vertical length of the stenosis, which represents a very important parameter to choose the best surgical procedure, we performed a videotracheoscopy in all of the patients with a diagnosis of subglottic stenosis.

\section{Results}

A total of 19 patients were included in the study; 9 of them were male (47.3\%), and 10 were female (52.6\%). The age at diagnosis ranged between 19 and 68 years old, with a mean age of 46.47 years old. A total of 13 patients (68.4\%) suffered pulmonary involvement and 12 (63.1\%) suffered renal involvement 


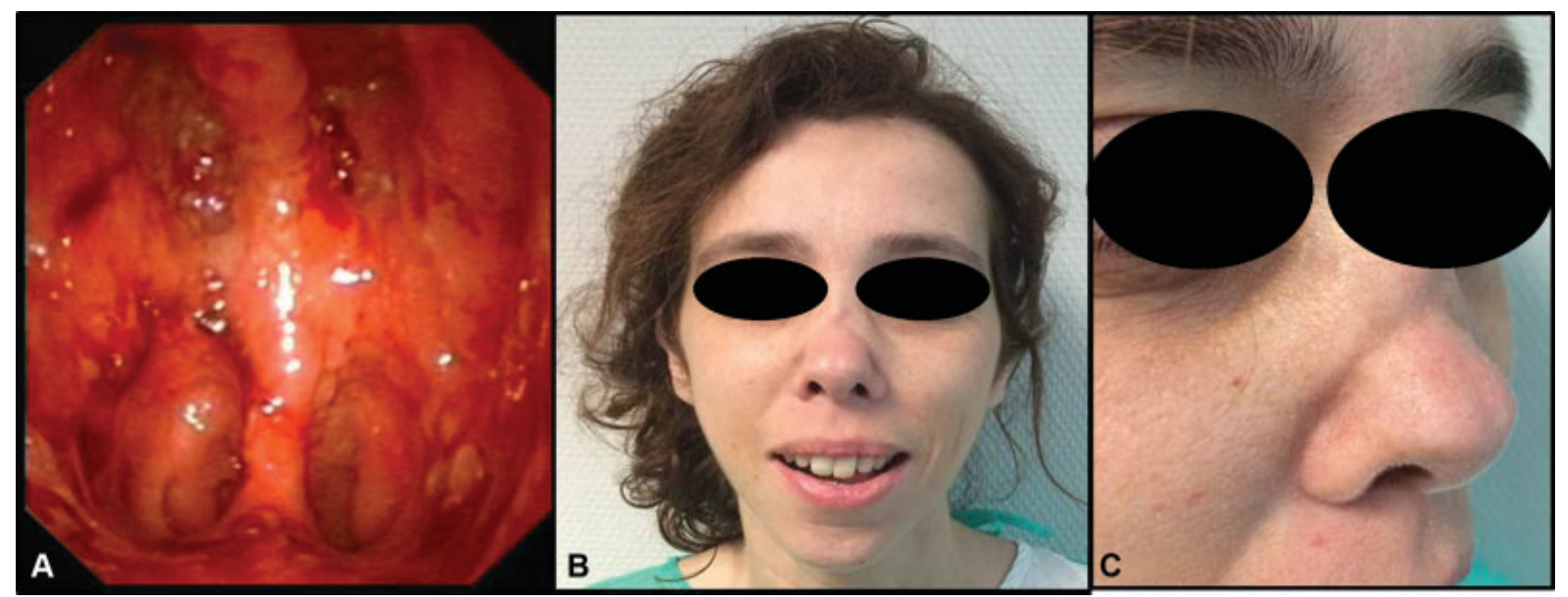

Fig. 2 Nasal septal necrosis and saddle nose deformity. (A) Endoscopic view of nasal cavity. (B) Saddle nose deformity: frontal view. (C) Saddle nose deformity: lateral view.

during the course of the disease. Head and neck manifestations were present in 16 of the 19 patients (84.2\%). Nasal involvement was the most common ear, nose and throat(ENT) manifestation, affecting 9 of 16 patients (56\%), followed by laryngotracheal involvement, which was diagnosed in 5 of 16 patients (31.25\%), and ear involvement in 4 of 16 patients (25\%). In 7 patients, the ENT symptoms, specifically sinonasal symptoms, were the first manifestation of the disease (43.75\%). Among the patients with nasal and sinonasal symptoms (9 patients), nasal obstruction and rhinorrhea were the most frequent symptoms, affecting 6 (66.6\%) and 4 (44.4\%) patients, respectively. Of these, 3 had sinuses involvement (33.3\%), and another 2 patients (22.2\%) had epistaxis and nasal crusts with severe pain and septal perforation with saddle nose deformity ( - Fig. 2). Sinonasal symptoms

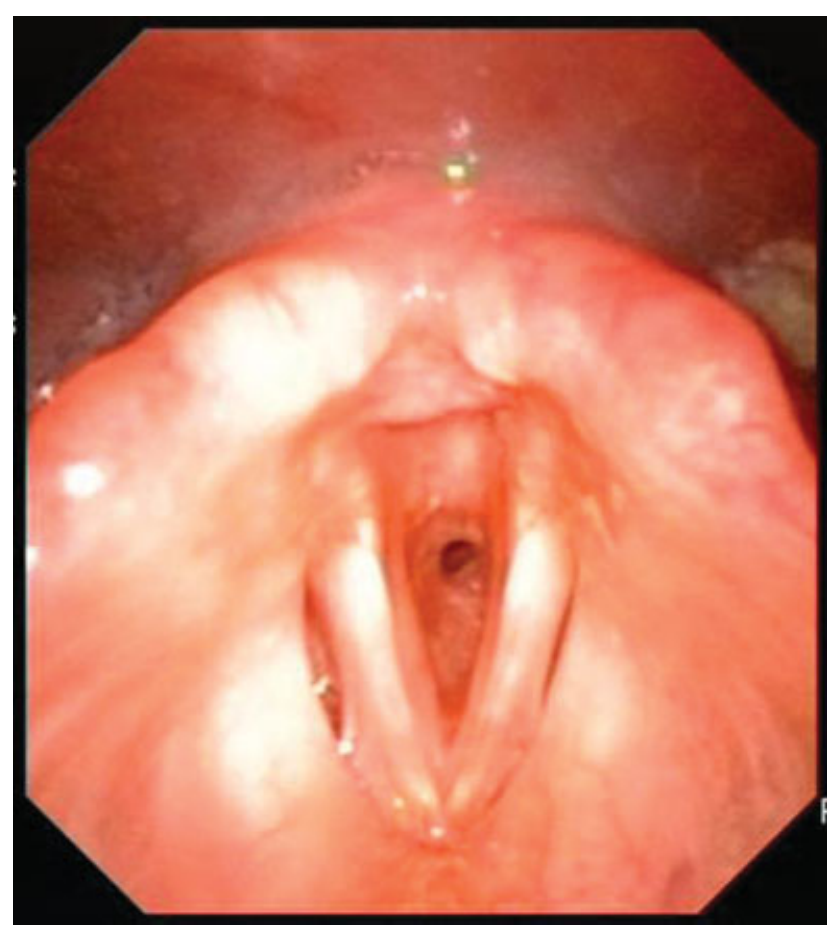

Fig. 3 Subglottic stenosis: endoscopic view. were the first manifestation of GPA in 3 of 16 patients (18.75\%); in other 4 patients, it was the only manifestation of GPA. Among the patients with laryngotracheal involvement, dyspnea was the most common symptom, caused by subglottic stenosis in 5 patients (31.25\%) (-Fig. 3). Four of them were classified as grade 1 stenosis (Myer-Cotton classification), and 1 as grade 2 (70\% of stenosis), all of them with no more than $1 \mathrm{~cm}$ in vertical length. None of them needed an emergency tracheostomy procedure. This procedure should be avoided, if possible, because of the risk of formation of excessive scar tissue that could make a future tracheal resection and reconstruction very challenging. All of the cases were treated with several endoscopic balloon dilations and laser $\mathrm{CO} 2$ resections, showing acceptable functional results, with the exception of the patient with grade 2 stenosis, who showed a worsening of symptoms and an excessive scar formation after two endoscopic laser $\mathrm{CO} 2$ procedures and balloon dilations. Finally, this patient was classified as presenting with grade 3 stenosis (90\%) and underwent a cricotracheal resection with end-to-end anastomosis (-Fig. 4). Currently, after a 4-year follow-up, the patient is asymptomatic, showing a good airway patency in the laryngeal videoendoscopy. The first symptom of this patient was nasal obstruction, with septal crusts and progressive nasal septal necrosis that caused a saddle nose deformity, which was treated by a rhinoseptoplasty with rib cartilage. Among the other four patients, two died from other causes and two maintained an acceptable airway pathway during a follow-up of 5 and 7 years, needing an endoscopic balloon dilation/Laser $\mathrm{CO} 2$ resection in 2 and 3 occasions, respectively. Among the patients with otologic involvement, we had two cases of serous otitis that were treated by the placement of tympanic ventilation tubes, and other two cases with sudden sensorineural hearing loss, which improved with systemic and intratympanic steroid treatment. We had no cases of middle chronic otitis media or facial palsy. All of the patients were treated with immunosuppressants and corticosteroids. Four patients underwent surgery: we performed two rhinoseptoplasties with rib cartilage reconstruction, one cricotracheal resection with end-to-end anastomosis, and two myringotomies with the placement of tympanic tubes. In three 


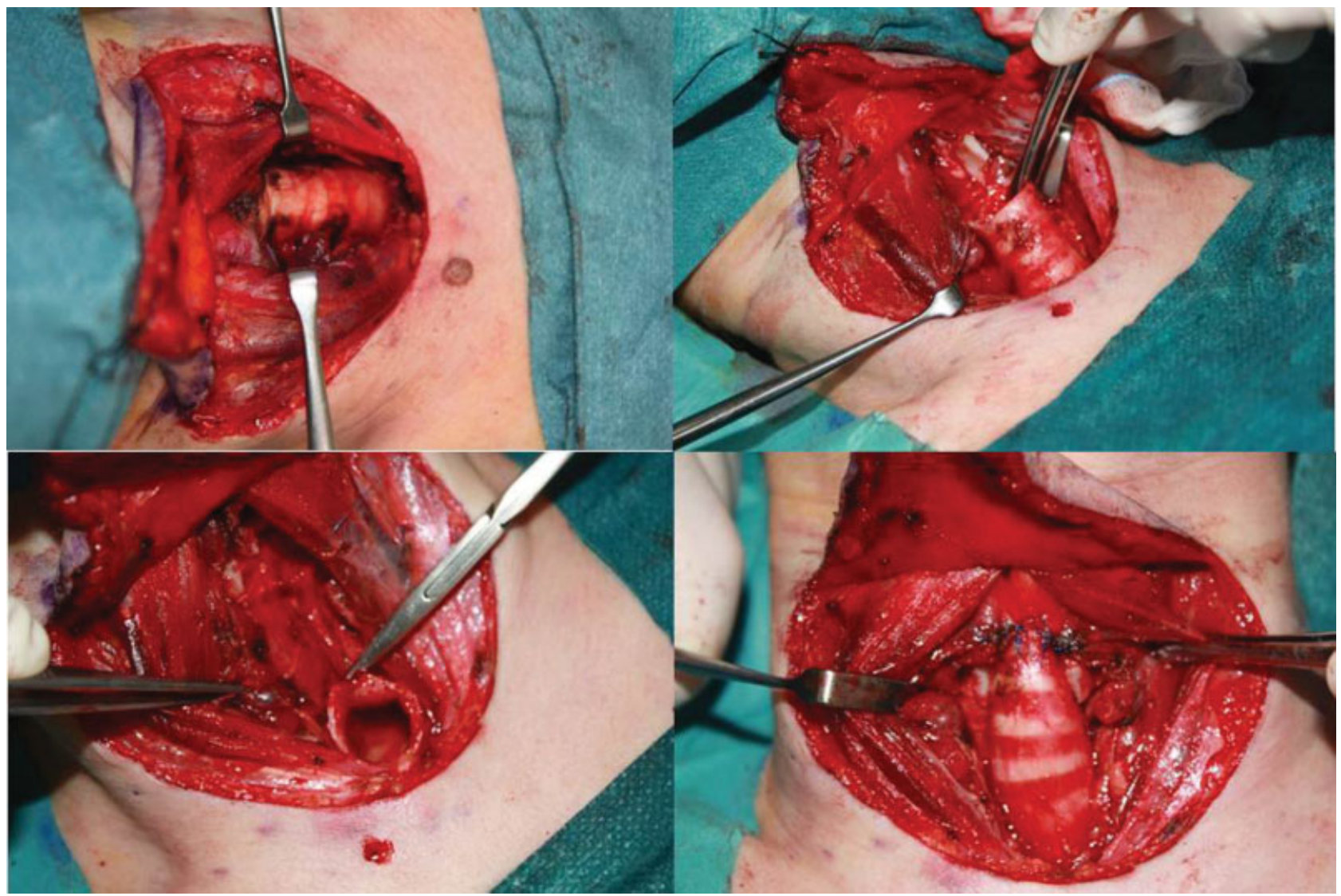

Fig. 4 Subglottic stenosis. Surgical reconstruction.

cases in which sinonasal symptoms were the first clinical manifestation, and in another four cases in which sinonasal symptoms were the only manifestation of GPA, a biopsy of the nasal mucosa was performed. It confirmed the histological criteria of GPA in five of them. In two patients with a negative biopsy, the indirect immunofluorescence showed a c-ANCA pattern. The other nine patients were referred to the ENT department to receive treatment for their head and neck symptoms after the diagnosis of GPA in other departments.

\section{Discussion}

The clinical course of GPA can be rapid or indolent. Constitutional signs and symptoms such as fever, weight loss, and fatigue, are common, but rarely dominate the clinical picture. The diagnosis is based on clinical manifestations suggestive of the disease (head and neck, pulmonary, and renal disease), and is confirmed by compatible histologic findings: systemic vasculitis affecting mainly small vessels, and necrotising granulomatous inflammation with giant multinucleate cells. These findings may be present together or isolated. Clinical suspicion of GPA should be considered in patients with unexplained refractory head and neck symptoms and systemic complaint. Usually, GPA involves otolaryngologic manifestations; more than $70 \%$ of the presenting symptoms take the form of nasal, sinus, ear, or tracheal manifestations. Upper respiratory tract involvement generally precedes pulmonary or renal involvement.
Pulmonary involvement ranges from asymptomatic pulmonary nodules to pulmonary infiltrates and fulminating alveolar hemorrhage. The most common renal involvement is necrotising segmental glomerulonephritis, though proliferative glomerulonephritis is also frequently present. Although the incidence of renal manifestations at the initial diagnosis of GPA is of only $18 \%$, > 75\% of the patients will ultimately develop kidney involvement. The range of other organ systems that may be involved in GPA is vast. Musculoskeletal system involvement may take the form of myalgias, arthralgias and arthritis. Cutaneous lesions include palpable purpura, ulcers, vesicles, papules and subcutaneous nodules. While rare, cardiac involvement may include pericarditis and valve lesions. ${ }^{10}$ Neurologic symptoms may arise in the form of mononeuritis multiplex, neuropathy, stroke, seizure, cerebritis or meningitis. Generalized symptoms, such as fever and malaise, may also be present.

The presence of ENT involvement in patients with GPA ranges between 72.3 and $99 \%$ of the cases, depending on the performance or not of systematic head and neck studies upon suspicion or diagnosis of disease, as well as periodic reassessment of new outbreaks. ${ }^{11}$ In our series, $84.2 \%$ of the patients (16 out of 19) showed ENT manifestations during the course of their disease, with sinonasal symptoms being the most frequent ones.

In between 63 and $72 \%$ of the cases, ENT symptoms are the first manifestation of GPA, ${ }^{12}$ although in our study they were the first symptoms in only $43.75 \%$ of the patients. 
The nose and paranasal sinuses are involved in up to $80 \%$ of GPA cases. Involvement can vary from mild obstruction to nasal collapse. ${ }^{13}$ Sinonasal involvement in GPA is often misdiagnosed in its early stages as chronic rhinitis or sinusitis. Nasal signs and symptoms include mucosal edema with obstruction, rhinorrhea, ulcerations, crusting, and epistaxis. Chronic sinusitis affects between 40 and $50 \%$ of the patients with sinonasal disease. ${ }^{13}$ Osteocartilaginous destruction with septal perforation and saddle nose deformity does not correlate closely with the active disease. A physical examination usually reveals the presence of nasal crusts and, occasionally, perforation of the anterior nasal septum. These lesions are often caused by vasculitis of the Kiesselbach area. In our series, nasal and paranasal symptoms turned out to be the most common ENT manifestation of GPA, affecting $\sim 56 \%$ of the cases (9/16), with sinuses involvement confirmed by a computed tomography (CT) exam in 3 patients. Two patients presented with saddle nose deformity due to necrosis of the septal cartilage and underwent a septorhinoplasty with autologous rib cartilage.

Otologic involvement occurs in between 25 and $40 \%$ of the patients during the course of GPA. Otitis media occurs in between 40 and $70 \%$ of the cases with otologic involvement. Otitis media may be the presenting feature and sole manifestation of GPA. Serous otitis media is the most prevalent type and is usually secondary to associated nasal disease and subsequent eustachian tube dysfunction. Up to $30 \%$ of the patients with GPA require a tympanostomy during the course of the disease. ${ }^{14}$ Granulomatosis with Polyangiitis may cause a direct involvement of the middle ear and/or of the mastoid mucosa in $\sim 10 \%$ of the cases, with necrotising granulomas and vasculitis that can result in persistent conductive hearing loss. Involvement of the external ear is rare, with external otitis or auricular chondritis. After renal insufficiency, sensorineural hearing loss is the most common long-term morbidity associated with GPA, affecting 35\% of the patients. ${ }^{15}$ Hearing loss may develop rapidly, progressing over days to weeks, being compatible with the profile of typical autoimmune hearing loss. Its cause is unclear: the suggested mechanisms include cochlear nerve compression by adjacent granuloma, cochlear immune-complex deposition, and local vasculitis involving the cochlear vessels. In our series, middle ear involvement was diagnosed only in two patients, who required tympanostomy tube insertion, due to serous otitis media (12.5\%). It is possible that the true incidence of middle ear involvement has not been sufficiently considered in our retrospective study, and that patients with this type of symptoms were treated in a primary care center. We had no cases of chronic otitis media, facial palsy or external ear involvement, but diagnosed two cases of sudden bilateral sensorineural hearing loss, which were treated with the administration of systemic steroids associated with intratympanic dexamethasone injections.

Oral and oropharyngeal involvement are relatively rare, affecting $6 \%$ of the cases, although a classic occurrence is that of 'strawberry' gingival hyperplasia. Deep mucosal ulcerations of the oropharyngeal mucosa may also be observed, but they may be nonspecific for GPA. ${ }^{16}$
The manifestations of laryngotracheal involvement in GPA may range from subtle hoarseness to stridor and life-threatening obstruction, with subglottic stenosis being the most common, representing the first manifestation of the disease in between 1 and $6 \%$ of the cases. Subglottic stenosis is more common when the diagnosis of GPA takes place in patients $<20$ years old. It occurs in between 16 and $20 \%$ of all patients with GPA and in up to $50 \%$ of the pediatric patients with GPA, ${ }^{17}$ and can be the only manifestation of GPA.

Patients with subglottic or tracheal stenosis may be asymptomatic in the initial phases of the disease or may suffer dyspnea with exercise, but sometimes they may eventually develop acute respiratory obstruction. The immediate subglottic region of the trachea is particularly susceptible to narrowing due to laryngopharyngeal reflux, limited blood supply, and turbulent airflow. This narrowing of the upper airway is associated with scarring and can result in airway compromise. Chronic scarring is thought to be the result of a recurrent insult, such as laryngopharyngeal reflux in the presence of GPA, rather than of only an ongoing microvasculitis. Only $20 \%$ of the involved cases diminish with immunotherapy; $80 \%$ remain fixed or irreversible, because of chronic fibrosis. Treatment includes intralesional corticosteroids, tracheotomy, or surgical reconstruction. Methylprednisolone acetate injection combined with the serial passage of blunt dilators has proven to be another effective way of managing treatment-refractory subglottic stenosis. ${ }^{17}$

In our series, five patients were diagnosed with some degree of subglottic stenosis. All were treated by endoscopic surgery, and in one patient a laryngotracheal reconstruction was performed. As explained above, we usually try as much as possible to avoid a tracheostomy procedure, since we consider that it can increase the vertical length of the stenotic segment through the production of fibrous tissue. This makes a tracheal anastomosis particularly difficult, should it be required in the future. No single patient of our study group required a tracheostomy. The patient with grade 2 stenosis underwent a cricotracheal resection with end-to-end reconstruction, after having shown a bad evolution as a consequence of more conservative procedures. Our cricotracheal technique considers local anesthesia with sedation and spontaneous breathing, because the absence of the orotracheal tube makes the intraoperative evaluation of the stenotic segment that must be resected significantly easier. In fact, while the main surgeon performs the surgical approach of the neck, exposing the subglottic area and the upper trachea, another member of our group carries out a laryngeal videoendoscopy to identify the stenotic segment. Since the light of the flexible endoscope may be seen through the thin tracheal wall, it helps us to evaluate the exact segment that requires a resection.

Ocular abnormalities, such as orbital pseudotumor, episcleritis, and peripheral ulcerative keratitis have been noticed in almost $50 \%$ of the patients at some point in the course of the disease. Permanent visual loss has been detected in up to $8 \%$ of the patients as a sequela to the active disease, usually resulting from retro-orbital disease and subsequent optic nerve ischemia. ${ }^{15}$ In our series, we did not find patients with ocular symptoms related to GPA. 
A definitive diagnosis can be made by a biopsy of suspicious lesions in conjunction with positive serologic analysis. Probably, biopsies of sinonasal lesions are the best possibility for diagnosis in the head and neck region, since they enable extensive sampling (generally, samples over $5 \mathrm{~mm}$ are required), thus facilitating the detection of histologically compatible lesions, but it may be necessary to repeat biopsies, because these lesions may be not diagnosed in up to $50 \%$ of the specimens. The biopsy of subglottic lesions should be undertaken with caution, as it can cause edema and further scarring. Alternative biopsy sites include the sural nerve, muscles, the skin, the lungs or the kidneys. ${ }^{18}$ Biopsy of granulation tissue in the middle ear is rarely diagnosed, because of the limited amount of sample taken for diagnosis, except for those performed in the context of mastoidectomy. Subglottic biopsies are not very useful, since it is also difficult to obtain large samples without causing subsequent scarring sequelae.

Laboratory testing for C-ANCA is an important component of the workup. Antineutrophil antibodies are targeted toward two main neutrophil granule components, serine PR3 and myeloperoxidase. Antibodies to PR3 are pathognomonic for GPA, whereas antibodies to myeloperoxidase are nonspecific (rheumatoid arthritis, ulcerative colitis, etc.). There are two methods to detect ANCA: immunofluorescence and enzyme-linked immunosorbent assay (ELISA). The first one is a more traditional method and it distinguishes the two different types of antibodies (anti-PR3 and antimyeloperoxidase) by the pattern of staining: cytoplasmic ANCA for anti-PR3 (c-ANCA), versus perinuclear ANCA (p-ANCA) for antimyeloperoxidase. A positive cytoplasmic ANCA test strongly suggests the diagnosis of GPA. The advent of ELISA testing has provided an additional means of discerning anti-PR3 from antimyeloperoxidase antibodies with enhanced specificity. When immunofluorescence and ELISA testing are combined, the sensitivity and specificity exceed $90 \%$ and $98 \%$, respectively. Nevertheless, there are potential pitfalls in interpreting ANCA results. False positive cytoplasmic ANCA results may be associated with diverse entities, such as cocaine use and HIV infection. In addition, $10 \%$ of the patients with GPA show a perinuclear pattern on immunofluorescence, and up to $20 \%$ of the patients with active, untreated GPA lack ANCA, a percentage that may increase to $30 \%$ in the limited form of the disease. In patients with suggestive clinical history, but negative cytoplasmic ANCAs, serial serologic testing may ultimately reveal a positive cytoplasmic ANCA test. In patients in whom GPA has been diagnosed, the cytoplasmic ANCA titer may be a barometer of disease activity, and a rise in cytoplasmic ANCA titer and/or in the level of anti-PR3 antibodies may be predictive of a flare or relapse of the disease. ${ }^{9}$

In our series, 7 of 16 patients were diagnosed at the ENT department; 5 of them by the performance of a positive biopsy of the nasal mucosa and of a positive ANCA study, and other 2 patients by the performance of a c-ANCA pattern, without confirmation at biopsy. Finally, $71.4 \%$ (5/7) of the nasal biopsies showed histological findings that confirmed the diagnosis of GPA.

The patient who underwent laryngotracheal reconstruction due to severe subglottic stenosis had been diagnosed by nasal mucosa biopsy before having dyspnea. The other patients came to the ENT department for treatment of head and neck symptoms after receiving the GPA diagnosis in different departments. Immunofluorescence was used in all of the patients at the time of diagnosis and also in the follow-up in order to plan the nasal surgery in a non-active phase of the disease. Among 16 patients that underwent the ANCA study, 14 were positive; 11 cases were positive for anti-PR3 with a cytoplasmic pattern (c-ANCA), and 3 were positive for myeloperoxidase with a perinuclear pattern (p-ANCA). The other two patients with negative indirect immunofluorescence only had sinonasal manifestations of the disease, and the diagnosis was performed by a biopsy of the nasal mucosa.

Performing tone audiometry is also useful in the initial evaluation of patients with GPA or suspected to suffer from it, since the development of bilateral sensorineural hearing loss throughout the evolution of the disease is not uncommon, as previously mentioned. ${ }^{1}$ Performing CT or magnetic resonance imaging (MRI) scans may be indicated in some patients, according to the location of the involvement and to the clinical manifestations. The most common findings on CT scans of the paranasal sinuses and fossae are the presence of mucosal edema with bone destruction foci in the paranasal sinuses, as well as foci of sclerosing osteitis and bone thickening in the same location. ${ }^{19}$ In severe subglottic/tracheal stenosis, CT and MRI scans may also help to evaluate the characteristics of the lesions. These tests are also indicated for patients with ear involvement who are unresponsive to treatment or in cases of facial paralysis.

A retrospective study by D'Anza et al examining sinonasal imaging findings (from CT scanning and magnetic resonance imaging MRI) in GPA suggested that although the imaging tests demonstrate nonspecific findings, the existence of septal erosion, mucosal thickening, and bony changes increase the probability of GPA. The study reported that mucosal thickening, bony destruction, and septal erosion occurred in $87.7 \%$, $59.9 \%$, and $59.4 \%$ of the patients, respectively. ${ }^{14}$

Surgical treatment does not change the course of the disease, but it can contribute to ameliorate the consequences of tissue destruction in the head and neck region. Surgery has to be avoided in the reactivation phases of the disease, because it can lead to greater sequelae. We have used serial measurements of c-ANCA titers to follow the evolution of the disease, before deciding the time of nasal reconstruction. We have performed two septorhinoplasties for saddle nose deformity, with good results and without complications. Among the patients with laryngotracheal involvement, only $20 \%$ responded to medical treatment; in our series, we have performed endoscopic surgery for all five patients with subglottic stenosis and a cricotracheal resection with end-to-end anastomosis in a more severe case.

\section{Conclusions}

Head and neck symptoms represent the most frequent clinical manifestations of GPA, and they can be either the first or the only symptom. Patients with exclusive sinonasal or laryngotracheal symptoms are not uncommon. The otolaryngologists play a central role in the diagnosis and in the 
long-term treatment of these patients and have to keep this pathology in mind in patients with ENT symptoms that do not respond to the treatment. The close collaboration with rheumatologist colleagues is essential for an effective medical treatment of these patients. Surgery may be, in fact, a good option in selected patients, because it can contribute to improve their quality of life.

\section{References}

1 Holle JU, Laudien M, Gross WL. Clinical manifestations and treatment of Wegener's granulomatosis. Rheum Dis Clin North Am 2010;36(03):507-526

2 Cadoni G, Prelajade D, Campobasso E, et al. Wegener's granulomatosis: a challenging disease for otorhinolaryngologists. Acta Otolaryngol 2005;125(10):1105-1110

3 Cummings CW, Haughey BH, Thomas JR, et al. Otolaryngology Head and Neck Surgery. 4th ed. St. Louis, MO: Mosby, Inc; 2005: 934-936; 1493-1508.

4 Klippel JH. Primer on the Rheumatic Diseases. 12th ed. Atlanta, GA: Arthritis Foundation; 2001:392-394, 643.

5 Watts RA, Lane SE, Scott DG, et al. Epidemiology of vasculitis in Europe. Ann Rheum Dis 2001;60(12):1156-1157

6 González-Gay MA, García-Porrúa C. Epidemiology of the vasculitides. Rheum Dis Clin North Am 2001;27(04):729-749

7 Popa ER, Tervaert JW. The relation between Staphylococcus aureus and Wegener's granulomatosis: current knowledge and future directions. Intern Med 2003;42(09):771-780

8 Jennette JC, Xiao H, Falk RJ. Pathogenesis of vascular inflammation by anti-neutrophil cytoplasmic antibodies. J Am Soc Nephrol 2006;17(05):1235-1242
9 Erickson VR, Hwang PH. Wegener's granulomatosis: current trends in diagnosis and management. Curr Opin Otolaryngol Head Neck Surg 2007;15(03):170-176

10 Seo P, Stone JH. The antineutrophil cytoplasmic antibody-associated vasculitides. Am J Med 2004;117(01):39-50

11 Morales-Angulo C, García-Zornoza R, Obeso-Agüera S, Calvo-Alén J, González-Gay MA. [Ear, nose and throat manifestations of Wegener's granulomatosis (granulomatosis with polyangiitis)]. Acta Otorrinolaringol Esp 2012;63(03):206-211

12 Srouji IA, Andrews P, Edwards C, Lund VJ. Patterns Granulomatosis. ENT aspects. J Laryngol Otol 2007;121:653-659

13 Gubbels SP, Barkhuizen A, Hwang PH. Head and neck manifestations of Wegener's granulomatosis. Otolaryngol Clin North Am 2003;36(04):685-705

14 D'Anza B, Langford CA, Sindwani R. Sinonasal imaging findings in granulomatosis with polyangiitis (Wegener granulomatosis): A systematic review. Am J Rhinol Allergy 2017;31(01):16-21

15 Hoffman GS, Kerr GS, Leavitt RY, et al. Wegener granulomatosis: an analysis of 158 patients. Ann Intern Med 1992;116(06): 488-498

16 Rasmussen N. Management of the ear, nose, and throat manifestations of Wegener granulomatosis: an otorhinolaryngologist's perspective. Curr Opin Rheumatol 2001;13(01):3-11

17 Gluth MB, Shinners PA, Kasperbauer JL. Subglottic stenosis associated with Wegener's granulomatosis. Laryngoscope 2003;113 (08):1304-1307

18 Congdon D, Sherris DA, Specks U, McDonald T. Long-term follow-up of repair of external nasal deformities in patients with Wegener's granulomatosis. Laryngoscope 2002;112(04):731-737

19 Benoudiba F, Marsot-Dupuch K, Rabia MH, Cabanne J, Bobin S, Lasjaunias P. Sinonasal Wegener's granulomatosis: CT characteristics. Neuroradiology 2003;45(02):95-99 